\title{
Migraine Literacy and Treatment in a University Sample
}

\author{
Stephanie C. Goodhew ${ }^{1}$ (I)
}

Accepted: 2 August 2019/Published online: 13 August 2019

(C) The Author(s) 2019

\begin{abstract}
There are many factors that can lead to underutilization of appropriate medical treatments for migraine, including a poor understanding regarding the diagnostic features of migraine and available treatment options. The purpose of the present study was therefore to assess migraine literacy and factors leading to different treatment decisions. Respondents were 229 university students, both migraineurs and non-migraineurs, who completed online questionnaires which surveyed their knowledge of migraines, and for migraineurs, their experiences of migraine and migraine treatment. While migraineurs had significantly greater migraine literacy than non-migraineurs according to the questions asked, knowledge was incomplete in both groups. The majority of migraineurs who used prescription medication for acute pain relief found such medications to be very effective, and for those who did not use such medications, one in five cited the reason was that they did not know that such medications were available. The imperfect migraine literacy, both in terms of diagnostic features and available treatments, highlights avenues for future public health awareness campaigns.
\end{abstract}

Keywords Migraine $\cdot$ Migraine knowledge $\cdot$ Migraine literacy $\cdot$ Health literacy $\cdot$ Migraine treatment

\section{Introduction}

Despite the suffering and disability that migraine conditions bring, migraine is often underdiagnosed and undertreated in clinical settings [1-3]. One key reason for this may be a poor understanding in the general public of the diagnostic features of migraine, in particular, the symptoms that facilitate differential diagnosis of migraine versus non-migraine headache.

\section{Research Highlights}

- Found higher rates of migraine literacy among migraineurs than nonmigraineurs

- Identified key areas of incomplete migraine literacy regarding both diagnosis and treatment that should be targeted in future public health awareness campaigns

This article is part of the Topical Collection on Medicine

Electronic supplementary material The online version of this article (https://doi.org/10.1007/s42399-019-00124-y) contains supplementary material, which is available to authorized users.

Stephanie C. Goodhew

Stephanie.Goodhew@anu.edu.au

1 The Research School of Psychology, The Australian National University, Building 39, Canberra, Australian Capital Territory 2601, Australia
For example, migraines are often mistaken for sinus headaches $[4,5]$. However, best-practice management of migraines typically requires migraine-specific treatments, such as triptans $[6,7]$. Therefore, if patients presenting in clinical settings are not aware of the defining differences between migraine and non-migraine headache, patients may not articulate these symptoms or request appropriate treatment, then some of the shortcomings of treatment in clinical settings could stem from these issues. The goal of the present study was therefore to examine the role of migraine literacy in a non-clinical population. That is, if migraine literacy is limited, future interventions aimed at raising awareness of the diagnostic features of migraine and available treatments could improve clinical outcomes if patients understand how their symptoms relate to migraine diagnosis and available treatments, and are therefore better placed to advocate for their own care.

In the present study, migraine literacy and other potential barriers to full utilization of effective treatment were assessed in a university student sample. A university sample is, by definition, a highly educated sample. This means that this group likely represents one of the maximum levels of literacy: any deficits of knowledge observed in this sample are only likely to be accentuated in other groups. Therefore, the present study examined migraine literacy and treatment experiences and decisions in a university sample. 


\section{Method}

Respondents were recruited to complete a Health Questionnaire via an electronic participation website, where students enrolled in eligible courses were offered course credit in exchange for participation. There were no eligibility criteria for participation imposed. The questionnaire was administered online via Qualtrics. All provided informed consent prior to participation and all aspects of the study were approved by the Australian National University (ANU) Human Research Ethics Committee (Protocol number 2018/034). The sample size was not prescribed; instead, it was determined by the number of participants who volunteered throughout the semester that the study was available. The semester that the study was available was semester 1, 2018 (FebruaryJune 2018).

The questionnaire was divided into three sections: two demographic items were included to characterize the sample, then the first major block assessed participants' general literacy and knowledge regarding migraines, and the second major block collected information about their own experiences of migraines and treatments. The first demographic item asked, "What gender do you identify as?", and the response options were male, female, and other. The second asked, "What is your age (in years)?", a numeric response (between 0 and 100) was requested.

Block 1: Migraine Knowledge These questions were designed to assess participants' knowledge of the defining features of migraine headache, with a particular focus on differentiating it from non-migraine headache. Questions 1-5 (Q1-Q5) made a statement, and participants were asked to indicate whether they thought it was True or False. For example, Q1 reads "Migraines have the same symptoms as other headaches." The full questionnaire and its response format can be found in Appendix A. The facts about migraine for these and other questions were obtained from the World Health Organization [8]. Note that the correct answers constitute a mix of true and false, so that a response bias toward true/false could not systematically affect the accuracy of responses to these items.

Q6-Q9 and Q11-Q14 asked to what extent a series of characteristics were associated with migraine (e.g., nausea, seizure, aura, for full list; see Appendix A). The response options were as follows: Is a defining characteristic of migraine (a headache would not be considered a migraine without this feature)/Is commonly associated with migraine/Is not commonly associated with migraine/Is never associated with migraine. For the purposes of scoring, the first two responses were treated as equivalent (i.e., associated with migraine) and the second two responses were treated as equivalent (i.e., not associated with). Q15 asked how long a migraine episode or attack typically lasts, from the following options: Seconds to minutes, Minutes to Hours, Hours to several days, and Days to weeks. Q10 and Q16 were control response check questions. Q17-Q19 probed participants' awareness about the availability of different migraine treatments. They asked a series of statements about medication for migraine requiring true/false responses. For example, Q17 stated "Prescription medications (i.e., those that require a script from a doctor) are available, which can work in the short term to relieve migraine symptoms."

Block 2: Migraine and Treatment Experience The second major block of questions asked participants about their experiences of migraine (if any) and treatments that they had used, and their reasoning and decision-making surrounding treatment options (see Appendix A for full list of questions). In particular, the goal was to identify common barriers to accessing effective treatment. In this block, Q1 asked, "Have you ever had a migraine headache?", with the response options Yes, No, and Have had a headache but do not know whether it was a migraine available. All of the following questions were asked if and only if respondents selected Yes or Do not Know to Q1. Q2 asked, "Have you been diagnosed with migraine by a health professional (e.g., your doctor or a specialist)?" The response options for this question were Yes and No. Next, participants were presented with this information and instruction on-screen: "For the following questions, if you have ever had a migraine headache, then please answer the following questions in relation to your migraine headaches. If you have had a headache but are unsure whether it was a migraine, then please answer the following questions in relation to your headaches."

Q3-Q8 were drawn from the Migraine Disability Assessment (MIDAS) questionnaire [9] and assessed the frequency of migraine experience and the resulting disability. For instance, Q3 asked, "On how many days in the last 3 months did you miss work or university because of your headaches?", while Q7 asked, "On how many days in the last 3 months did you have a headache (If a headache lasted more than 1 day, count each day)". Q9-Q17 collected information about respondents' experience with treatments for their headaches. Q9 asked, "When you have a migraine attack, do you use over-the-counter (i.e., purchased from a pharmacy or other store without a script from a doctor) medications to relieve your migraine)?" The response options were Yes and No. If respondents answered $N o$ to Q9, then their reasons for not accessing such medications was assessed, whereas if they answered Yes to Q9, then their experience of the treatment efficacy was probed. Similarly, Q12 asked, "When you have a migraine attack, do you use prescription medication (for which a script from a doctor is required) to relieve your migraine?". The response options were Yes and No. Respondents were then asked either about the effectiveness of this medication (if they responded Yes), or the reasons why they had not accessed such medications were probed if they answered No. 
Q15 asked, "Do you use prescription medication to reduce or prevent the recurrence of migraine attacks?'. The response options were Yes and No. If respondents answered Yes, then they were asked about the effectiveness of the treatment, whereas if respondents answered No, then they were asked to indicate why they had not accessed such treatments. Q18 asked, "Please select 'daily headache' below," where the response options were Monthly headache/Weekly headache/ Daily headache/Yearly headache. Q19 was an open-ended question that asked participants to share any other thoughts, reflections, or insights they would like about migraines that they would like.

Statistical Procedure Statistical analysis was conducted in Statistical Package for the Social Sciences (SPSS). The "Knowledge Metric" was computed as the mean accuracy of respondents' answers in each of the three groups across the 17 items in block 1 (excluding the two control check items) that assessed migraine-related knowledge. This Knowledge Metric variable was non-normally distributed (Shapiro-Wilk $p<0.001$ ), and therefore, non-parametric analyses were used to compare the groups (i.e., Kruskal-Wallis tests where three groups are compared and Mann-Whitney $U$ tests when two groups are compared). All of the statistical analyses were twotailed tests, where the critical alpha level was 0.05 .

\section{Data Availability}

Raw data are available here: https://osf.io/skqbh/.

\section{Results}

Screening and Demographics A total of 229 respondents completed the questionnaire. There were no missing data. There were three screening questions in the questionnaire. Three respondents were excluded from further analysis because they did not respond appropriately to the first two check questions (the first asked them to select the word "Migraine" from four possible response options, and a second asked participants to select "False" from the options true, neither true nor false, and false). Although a third question was also included in the final part of the questionnaire, it appeared that some respondents may have misread the question. It asked respondents to select "daily headache" from amongst four options (Monthly headache/Weekly headache/Daily headache/Yearly headache). In contrast to the first two questions, more than a few respondents answered this question incorrectly, despite providing detailed comments in subsequent sections, indicative of the fact that they were not unduly rushing their responses. Instead, since this question appeared amongst others asking individuals about their experiences of migraine, it seems likely that some of these respondents misread this question and were instead reporting how often on average they experienced headaches. Therefore, no respondents were excluded for not responding "Daily headache" to this question. The sample contained 172 females and 54 males, with a mean age of 20.03 years $(\mathrm{SD}=4.69)$. The respondents were classified into one of three groups: migraineur $(N=59)$, unsure $(N=92)$, and non-migraineur $(N=75)$ on the basis of their response to the question, "Have you ever had a migraine headache," where the response options were yes (= migraineur), Have had a headache but do not know whether it was a migraine (= unsure), and no (= non-migraineur). This means that $26 \%$ of respondents were definite migraineurs, $41 \%$ unsure, and $33 \%$ non-migraineurs.

Knowledge Assessment Seventeen questions were used to assess respondents' migraine and headache-related knowledge and compute the Knowledge Metric. The results are reported in Table 1. A Kruskal-Wallis test revealed that the migraineur, unsure, and non-migraineur groups scored significantly differently across this Knowledge Metric $(p<0.001)$. Subsequent Mann-Whitney $U$ tests revealed that migraineurs $(M=82 \%)$ scored higher than both non-migraineurs $(M=77 \%)$, $U=1591.00, p=0.005$, and the unsure group $(M=75 \%)$, $U=1658.00, p<0.001$, which did not reliably differ from one another, $U=3119.50, p=0.280$.

Migraineurs scored slightly higher than either of the other two groups in response to Q1 regarding whether migraines have the same symptoms as other headaches. For all groups, however, even for migraineurs, $20 \%$ of the sample were not correct on this point, and $24 \%$ and $32 \%$ of the non-migraineur and unsure groups, respectively. Respondents were reasonably accurate in their responses to Q2-Q4, whereas Q5 regarding the laterality of migraine pain produced lower accuracy. Chance-level performance (i.e., that which would be achieved purely by guessing) to these $\mathrm{T} / \mathrm{F}$ questions would be $50 \%$. For the non-migraineurs, accuracy was only $59 \%$ in response to this question, versus $61 \%$ and $70 \%$ for migraineurs. This highlights relatively low levels of knowledge regarding the fact that migraine is typically unilateral and that this can be a distinguishing feature from other headaches. The fact that nausea (Q6), sensitivity to light (Q8), sensitivity to sound (Q9), and pulsating headache (Q11) are associated with or defining features of migraine compared with other types of headache, whereas seizure (Q7) and loss of consciousness (Q12) are not, was all answered with a reasonable degree of accuracy (70-93\%). The association of sensory disturbances (often visual) such as aura with migraine (Q13) separated migraineurs (92\%) from non-migraineurs $(69 \%)$ and the unsure group $(65 \%)$ by over 20 percentage points.

All groups were below chance in their accuracy regarding the fact that migraine headache is often aggravated by a 


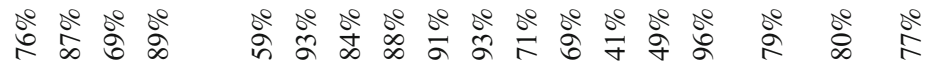

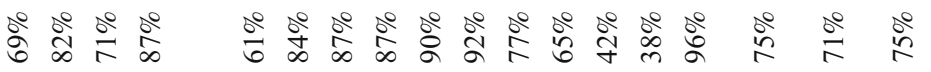

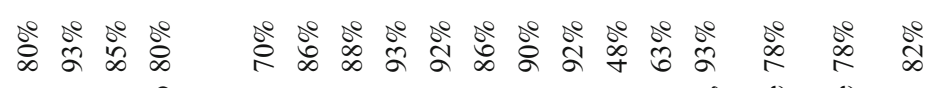


routine physical activity (Q14, 41-48\%). Accuracy was also low in response to Q15 regarding the duration of a typical migraine, with only $63 \%$ of migraineurs correctly identifying the hours to several days' duration, but the unsure group (38\%) and non-migraineurs (49\%) scored even lower. While the majority of migraineurs and non-migraineurs selected the correct answer, the next most common response for both groups was Minutes to Hours. This was also the most common response for the unsure group. This suggests that those who have had less experience with migraine or who feel less sure about its diagnostic features are likely to underestimate the duration of the average migraine attack. All groups showed a high level of awareness that prescriptions medications are available to offer short-term relief to migraine symptoms (Q17, 93-96\%). The awareness was somewhat lower regarding the potential dangers of medication overuse $(\mathrm{Q} 18$, 75-79\%) and the availability of prophylactic medication (Q19, 71-80\%).

Experience of Migraine and Migraine Treatment The questions in block 2 related to respondents' experience of migraine and migraine treatment. The results for these are shown in Table 2 (migraine experience) and Table 3 (treatment experience).

None of the unsure group had been diagnosed with migraine by a health professional (Q2), compared with $37 \%$ of the migraineur group. Q3-Q8 were from the MIDAS to assess migraine disability. The responses for the migraineur group versus the unsure group were compared statistically across these questions, and the migraineur group showed significantly higher levels of disability and pain than the unsure group $(p<0.05)$ according to Mann-Whitney $U$ tests for all except for the question regarding Q4. Despite the mean responses for the two groups being quite different on this question, there was considerable variability around the migraineur mean (note that SD is more than twice the mean) which would have contributed to this null result. This may be because making an assessment about how productivity is impacted is more difficult and varies across individuals to a greater extent than a question about how often one missed out on activities or suffered pain.

Just over half of the migraineurs used over-the-counter (OTC) medication to treat their pain, compared with just over a third of the unsure group. Of those who did use OTC medication, the majority of both migraineurs (58\%) and the unsure group ( $71 \%$ ) found them to be Moderately effective. The most commonly selected reasons for not using such medication for the migraineur group were My headaches are not severe enough to need them (36\%) and They do not relieve my migraine (29\%), and for the unsure group were My headaches are not severe enough to need them $(71 \%)$. A total of $11 \%$ of the migraineur group and $8 \%$ of the non-migraineur group were unaware that such medications were available. 


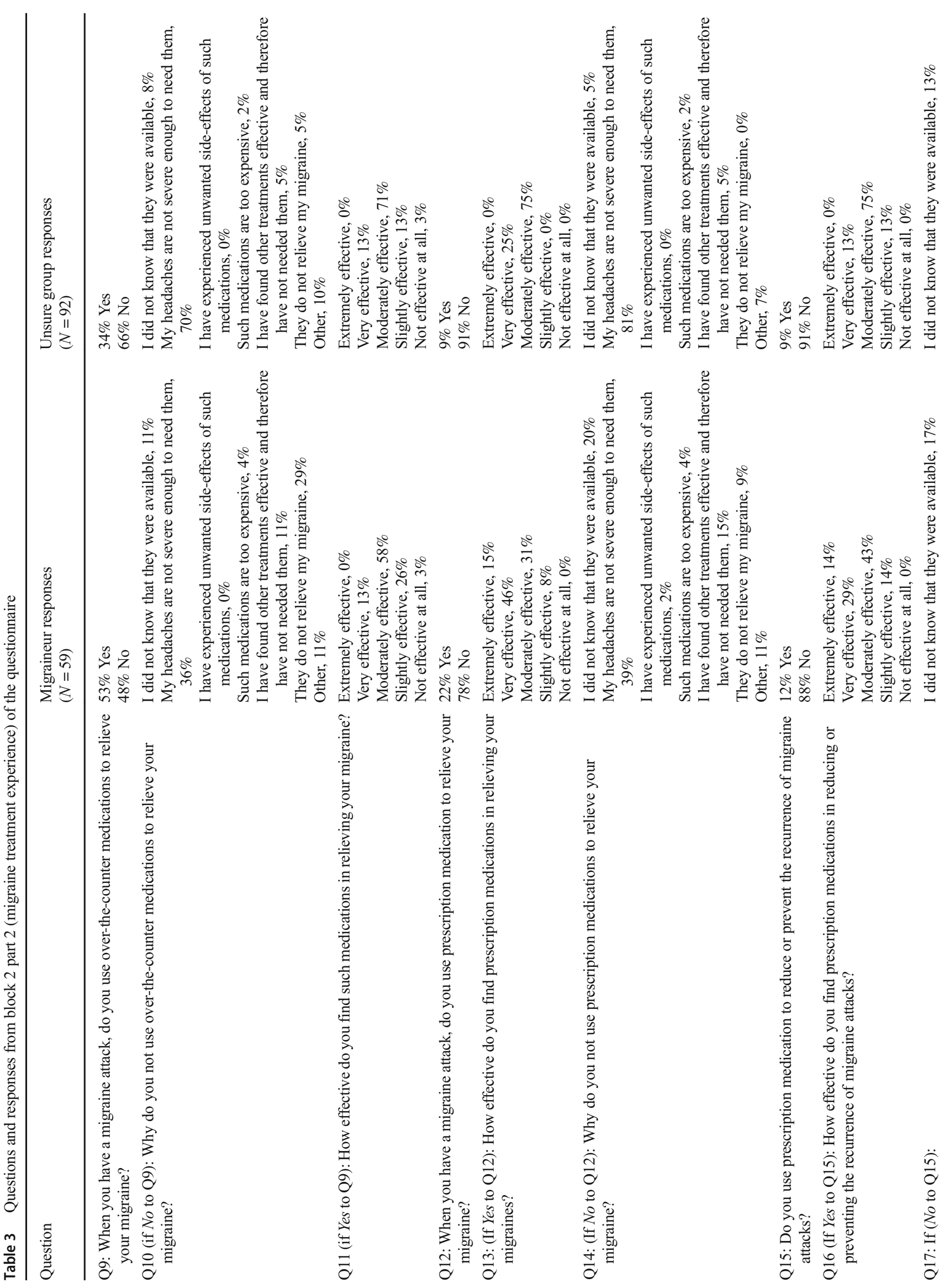


Only a minority of the migraineurs (22\%) and unsure group (9\%) used prescription medication to relieve their migraine attack. Of the migraineurs, the most commonly reported level of effectiveness of such prescription medications was Very effective (46\%), whereas for the unsure group, the most commonly reported level of effectiveness was Moderately effective $(75 \%)$. The most commonly reported reason for not using such medication amongst the migraineurs was My headaches are not severe enough to need them (39\%) and I did not know that they were available (20\%), compared with $M y$ headaches are not severe enough to need them (81\%) for the unsure group. Similarly, only a minority of migraineurs (12\%) and the unsure group (9\%) had used prescription medication to reduce or prevent the recurrence of migraine attacks. For migraineurs, the most commonly reported level of effectiveness of such medications was Moderately effective, for both the migraineur (43\%) and the unsure (75\%) groups. For those who did not use them, the most commonly reported reason for both groups (48\% and 73\%, respectively) was My headaches are not frequent enough to need them.

\section{Discussion}

Migraine is a disorder associated with considerable disability, suffering, and cost [10]. While promising new drugs are being developed [11], these do not address the substantial problems surrounding appropriate diagnosis and treatment of migraine that still remain. Therefore, there were two main purposes for the present study. First, this study sought to examine migraine literacy in a university sample, comparing individuals who had and had not experienced migraine headache. There were moderate levels of migraine literacy, and the questions asked significantly differentiated those with migraine from those who had not or were unsure about whether they had experience migraine headache. The fact that the migraineurs showed the highest levels of headache literacy likely stems from exposure to information from health professionals during the process of diagnosis and treatment, in addition to individuals' own research to understand the nature of their symptoms. That said, with average accuracy for the migraineurs and the other groups alike being well off $100 \%$. Approximately one in five migraineurs and one in four non-migraineurs were incorrect regarding whether headaches and migraines have the same symptoms. This is important because accurate diagnosis depends on differentiating migraine from other types of headache. This illustrates the need to more effectively disseminate information regarding the unique diagnostic hallmarks of migraine. For example, the fact that migraine pain is typically unilateral and aggravated by routine physical activity was not well understood, and the knowledge that migraine is associated with aura clearly separated those with migraine from the others in the sample. The finding that this university sample- 
by definition, a group with a high level of education - still lacked full knowledge regarding the identifying features of migraine suggests that, if anything, migraine literacy rates are likely to be even lower in the general population. This highlights opportunities for promoting greater health and headache literacy.

It was also concerning that approximately one in five migraineurs were not aware of the dangers of medication overuse. Overuse of acute pain-relief medications for migraine such as triptans leads to a form of chronic migraine known as medication-overuse headache which is a prevalent and widespread health problem [12]. The results of this questionnaire suggest that inadequate understanding of this danger may contribute to this problem. Therefore, there is a need for clearer and more effective dissemination of information around the dangers of medication overuse.

Second, the study was designed to examine individuals' experience of migraine and its treatment, with a view to identifying barriers to individuals accessing effective treatments. When it came to OTC treatments, some migraineurs felt that their headaches were not severe enough to warrant such treatments; however, another commonly cited reason was that they were not effective in relieving migraine. In contrast, the majority of the migraineurs who used prescription treatments for their pain found them to be very effective. This suggests that prescription medications best served migraineurs painmanagement needs when intervention was called for. However, of the migraineurs who did not use such treatments, one in five cited the reason being that they were unaware that such treatments were available. For all treatments, both acute and prophylactic, respondents only infrequently selected reasons such as side-effects and costs as barriers to treatment. Again, this highlights that there are improvements that can be made regarding public awareness of the availability and utility of such treatments to migraineurs, so that patients can better articulate their critical symptoms and advocate for their own care.

This study contributes to and extends upon the existing literature in important ways. Previous studies have revealed that migraine is often poorly understood and misdiagnosed in primary care settings [13, 14]. For instance, one study found that the majority of primary health care professionals were not aware that opioids can cause medication-overuse headache [13], thereby perpetuating and exacerbating the issue for which treatment is sought. Similarly, it is estimated that half of all migraineurs remain undiagnosed [15]. In contrast, improvements in patients' recognition of migraine-specific treatments have been documented after consultation with a specialist (i.e., neurologist) [16]. While this indicates that consultation with specialist healthcare professionals can be beneficial, it is also important to address general knowledge of migraine symptomology and treatment options, for example, via public health campaigns. This is because without this knowledge, there are significant potential barriers to individuals accessing the right care, which balances both the benefits and risks of different treatment approaches. If neither the patient nor primary health care professional recognizes migraines for what they are, then the patient is less likely to receive the appropriate care.

This study had some limitations which are important to acknowledge. First, the sample consisted of university students. This represents a highly educated sample, and therefore, if anything, may underestimate issues with migraine literacy. That is, migraine literacy may be even lower in the general population compared with this sample. However, a number of gaps were identified in migraine literacy here, and therefore, a general population sample would likely serve to further highlight the need for future interventions to target these knowledge gaps. Second, this study was advertised to potential volunteers as a Health Questionnaire. While no mention was made of migraine specifically, it is possible that this was of particular interest to individuals with health issues, including migraine. This may have elevated the number of migraineurs in the sample. Indeed, the rates of migrainereporting individuals (i.e., $26 \%$ definite migraineurs) are considerably above typical base rates for the condition in the general population, where estimates between 5 and $12 \%$ have been reported [17]. This could reflect that university students may be at elevated risk of migraine. This is plausible because university students are a high-risk group for psychological conditions $[18,19]$, which are often co-morbid with migraine [20]. Alternatively, it could simply reflect the fact that migraineurs (versus non-migraineurs) were disproportionately drawn to participate in this study. Third, this study relied on participants' self-report of their knowledge and experience. It would be useful to obtain converging evidence, for example, from health professionals regarding participants' diagnoses. That said, however, clinicians typically rely heavily on participants' self-report in both diagnosis (e.g., using the MIDAS or migraine diaries to assess frequency and severity) and treatment (e.g., determining whether a patient is satisfied with the efficacy of a particular treatment) [21,22]. At present, there is no objective measurement of migraine, and therefore, selfreport represents a reasonable method for research in this area.

\section{Conclusion}

In conclusion, this study identified some particular gaps and therefore avenues for future interventions to target regarding migraine symptomology and treatment literacy. In particular, public health campaigns should be targeted at improving awareness of some of the features that differentiate migraine, as well as both the availability but necessary precautions of medication options for migraine treatment. 
Authors' Contributions The sole author is responsible for all of the research.

\section{Compliance with Ethical Standards}

Ethics Approval and Consent to Participate This research was approved by the ANU. Human Research Ethics Committee (Protocol number 2018/034).

Consent for Publication Participants provided written informed consent to have their data included in scientific publications.

Competing Interests The author declares that she has no competing interests.

Open Access This article is distributed under the terms of the Creative Commons Attribution 4.0 International License (http:// creativecommons.org/licenses/by/4.0/), which permits unrestricted use, distribution, and reproduction in any medium, provided you give appropriate credit to the original author(s) and the source, provide a link to the Creative Commons license, and indicate if changes were made.

\section{References}

1. Gupta MX, Silberstein SD, Young WB, Hopkins M, Lopez BL, Samsa GP. Less is not more: underutilization of headache medications in a university hospital emergency department. Headache. 2007;47(8):1125-33.

2. Silberstein SD. Preventive migraine treatment. Continuum : Lifelong Learning in Neurology. 2015;21(4 Headache):973-89.

3. Sheftell FD, et al. Optimizing the diagnosis and treatment of migraine. JAANP. 2005;17(8):309-17.

4. Schreiber CP, Hutchinson S, Webster CJ, Ames M, Richardson MS, Powers C. Prevalence of migraine in patients with a history of selfreported or physician-diagnosed "sinus" headache. Arch Intern Med. 2004;164(16):1769-72.

5. Cady RK, Schreiber CP. Sinus headache or migraine? Considerations in making a differential diagnosis. Neurology. 2002;58(9 suppl 6):S10-4.

6. Thorlund K, Mills EJ, Wu P, Ramos E, Chatterjee A, Druyts E, et al. Comparative efficacy of triptans for the abortive treatment of migraine: a multiple treatment comparison meta-analysis. Cephalalgia. 2014;34(4):258-67.

7. Cameron C, Kelly S, Hsieh SC, Murphy M, Chen L, Kotb A, et al. Triptans in the acute treatment of migraine: a systematic review and network meta-analysis. Headache. 2015;55 Suppl 4:221-35.

8. Headache disorders. 2018 [cited 2018; Available from: http://www. who.int/en/news-room/fact-sheets/detail/headache-disorders. Accessed January 2018.

9. Stewart WF, et al. Development and testing of the Migraine Disability Assessment (MIDAS) questionnaire to assess headache-related disability. Neurology. 2001;56(6 Suppl 1):S20-8.
10. Pomes LM, Guglielmetti M, Bertamino E, Simmaco M, Borro M, Martelletti P. Optimising migraine treatment: from drug-drug interactions to personalized medicine. J Headache Pain. 2019;20:56. https://doi.org/10.1186/s10194-019-1010-3.

11. Do TP, Guo S, Ashina M. Therapeutic novelties in migraine: new drugs, new hope? J Headache pain. 2019;20:37. https://doi.org/10. 1186/s10194-019-0974-3.

12. Diener H-C, Limmroth V. Medication-overuse headache: a worldwide problem. Lancet Neurol. 2004;3(8):475-83.

13. Minen MT, Loder E, Tishler L, Silbersweig D. Migraine diagnosis and treatment: a knowledge and needs assessment among primary care providers. Cephalalgia. 2016;36(4):358-70.

14. Patwardhan MB, Samsa GP, Lipton RB, Matchar DB. Changing physician knowledge, attitudes, and beliefs about migraine: evaluation of a new educational intervention. Headache: J Head Face Pain. 2006;46(5):732-41.

15. Lipton RB, Diamond S, Reed M, Diamond ML, Stewart WF. Migraine diagnosis and treatment: results from the American Migraine Study II. Headache: J Head Face Pain. 2001;41(7):638 45.

16. Soon Y, Siow H, Tan C. Assessment of migraineurs referred to a specialist headache clinic in Singapore: diagnosis, treatment strategies, outcomes, knowledge of migraine treatments and satisfaction. Cephalalgia. 2005;25(12):1122-32.

17. MacGregor EA, Brandes J, Eikermann A. Migraine prevalence and treatment patterns: the global migraine and zolmitriptan evaluation survey. Headache: The Journal of Head and Face Pain. 2003;43(1): 19-26.

18. Ibrahim AK, Kelly SJ, Adams CE, Glazebrook C. A systematic review of studies of depression prevalence in university students. J Psychiatr Res. 2013;47(3):391-400.

19. Storrie K, Ahern K, Tuckett A. A systematic review: students with mental health problems - a growing problem. Int J Nurs Pract. 2010;16(1):1-6.

20. Antonaci F, Nappi G, Galli F, Manzoni GC, Calabresi P, Costa A. Migraine and psychiatric comorbidity: a review of clinical findings. J Headache Pain. 2011;12(2):115-25.

21. Lipton RB, Stewart WF, Sawyer J, Edmeads JG. Clinical utility of an instrument assessing migraine disability: the Migraine Disability Assessment (MIDAS) questionnaire. Headache: J Head Face Pain. 2001;41(9):854-61.

22. Steiner TJ, Jensen R, Katsarava Z, Linde M, MacGregor EA, Osipova $\mathrm{V}$, et al. Aids to management of headache disorders in primary care (2nd edition): on behalf of the European headache federation and lifting the burden: the global campaign against headache. J Headache Pain. 2019;20:57. https://doi.org/10.1186/ s10194-018-0899-2.

Publisher's Note Springer Nature remains neutral with regard to jurisdictional claims in published maps and institutional affiliations. 